\title{
THE RELATION OF NEUTRAL FAT TO LACTESCENCE OF SERUM 1
}

\author{
By MARGARET J. ALBRINK, EVELYN B. MAN, AND JOHN P. PETERS \\ (From the Department of Internal Medicine, Yale University School of Medicine, \\ New Haven, Conn.)
}

(Submitted for publication June 30, 1954; accepted October 6, 1954)

It has long been recognized that the cloudy or lactescent serum occurring in certain diseases contains an abnormally large amount of neutral fat (1), though a quantitative relationship between neutral fat and lactescence has not been established. In the present investigation the ultracentrifuge has been utilized to study the suspended lipid particles which are responsible for such lactescence. By analyzing a variety of clear and lactescent sera having high concentrations of one or more lipid components the relationship of neutral fat to lactescence has been verified and quantitatively defined.

\section{METHODS}

Serum lipids were determined before and after the removal of the suspended lipid particles by flotation in the ultracentrifuge. The technique of Van Eck, Peters, and Man (2) was modified as described below to permit the more complete separation of the lipids present as visible particles from the lipids present in clear solution. The particulate lipids which caused turbidity and were removed by centrifugation shall be referred to as "insoluble" lipid, while the lipids present in the clear subnatant after centrifugation shall be designated "soluble" lipid. The visual appearance of the serum was thus the criterion used in determining the state of solubility. No effort was made to classify the differential flotation characteristics of the lipids, the ultracentrifuge being used simply to remove the particles causing turbidity. Although no measurement of particle size was made, it is assumed that the insoluble lipids ranged from $0.1 \mu$ in diameter, the size just sufficient to scatter light (3), to $1 \mu$, the size of the larger chylomicrons.

Duplicate $5 \mathrm{ml}$. portions of undiluted serum, in no instance more than 24 hours old, were measured accurately into two $13.5 \mathrm{ml}$. lustroid tubes. The serum was spun for one hour at $18,000 \mathrm{rpm}$ (about 20,000 g.) at room temperature in a Spinco Model L Preparative Ultracentrifuge. After centrifugation the insoluble matter was visible on top as a buttery yellow layer in very lactescent serum, and as a faint white film in, clear or

1 Aided in part by a Grant from the United States Public Health Service and by an Institutional Grant from the American Cancer Society. slightly lactescent serum. The clear subnatant serum was removed from the bottom of each tube by piercing the "cream" layer with a No. 17, 2-1/2 inch needle, and inserting through this a long No. 20 needle to which was attached a Van Slyke pipette with a Luer-adapted tip. An exact quantity of the subnatant fluid was withdrawn into the pipette, leaving the "cream" layer floating undisturbed on top of the remaining serum. As the subnatant fluid was removed, exquisite care was taken to keep the tip of the outer needle just below the "cream" layer. In this way the fatty material which adhered to the outer needle was excluded from the clear subnatant fluid.

When the serum was moderately or grossly lactescent, the original serum and the clear subnatant fluid obtained after centrifugation were analyzed for lipids. The insoluble lipids were calculated by subtracting the lipids of the clear subnatant fluid from those of the original serum.

When the serum was clear or slightly lactescent and the anticipated difference between the original serum and the subnatant fluid was small, exactly $3 \mathrm{ml}$. of the subnatant fluid were removed from the bottom of the tube, and the "cream" layer was resuspended in the remaining $2 \mathrm{ml}$. of serum by gentle agitation of the lustroid tube. Any lipid adhering to the wall was scraped off and resuspended in the solution using the No. 17 needle as a stirring implement. This suspension was flushed in and out of the pipette-needle apparatus to insure complete mixing of the insoluble fat with the remaining subnatant fluid. In this way all the particulate lipid originally present in $5 \mathrm{ml}$. was concentrated in $2 \mathrm{ml}$. By subtracting the lipid values of the subnatant fluid from those of the "cream" suspension and multiplying the difference by two-fifths, the concentration of the insoluble lipids in the original serum was calculated. In the interests of uniformity all results were expressed as concentrations in the original serum (equal to the sum of the soluble and insoluble lipid) and in the clear subnatant, the difference between the two being the insoluble lipid. Although the differences appear small in the case of clear sera, they have added reliability because they are derived from the differences actually measured, which were over two times as great.

The accuracy of this method depends on the uniformity of distribution of lipids in the clear subnatant serum. Van Eck, Peters, and Man (2) found no consistent variation of lipids or protein of clear sera at different levels of the centrifuge tube. These findings have been essentially confirmed, with the exception that the 
protein was from 0.1 to $0.3 \mathrm{gm}$. per cent higher in the subnatant than in the original serum. This layering did not affect the lipids, which were the same at different levels of the subnatant fluid.

In heavily lactescent sera, the separation of the insoluble lipids was not quite complete, since there was a zone of diminishing lactescence extending about one-third or one-half of the way from the top buttery layer into the tube. Thus in one lactescent serum in which the lipids were determined in the top buttery disc and in three equal layers of the subnatant from top to bottom, the concentration of neutral fat in the original serum was calculated to be $85.6 \mathrm{mEq}$. per L. Assuming the bottom clear layer, in which the concentration of neutral fat was $19.5 \mathrm{mEq}$. per L., to be free of insoluble lipid, the insoluble neutral fat calculated by difference was $66.1 \mathrm{mEq}$. per L. Of this, $51.7 \mathrm{mEq}$. were in the top buttery disc, $10.3 \mathrm{mEq}$. in the layer immediately under the disc and $4.1 \mathrm{mEq}$. in the middle layer. Cholesterol and phospholipids were similarly concentrated in the top. Therefore, in moderately or greatly lactescent sera the subnatant sample was taken from the bottom clear zone, and the insoluble lipids calculated as the difference between the original serum and the bottom-most layer. Any small error introduced by the incompleteness of the separation was thus minimized.

The lipids, which were determined by methods previously described (4), included total titratable fatty acids after hydrolysis of the lipids, total cholesterol and in many cases free cholesterol, and lipid phosphorus. Neutral fat fatty acids were calculated indirectly. ${ }^{2}$

2 Neutral fat fatty acids were calculated from the following formula (4):

Neutral fat fatty acids in $\mathrm{mEq}$. per liter $=$ total fatty acids - (cholesterol fatty acids + phospholipid fatty acids), where cholesterol fatty acids in $\mathrm{mEq}$. per liter $=$ $10 \times$ esterified cholesterol (mg. \%) 386

and phospholipid fatty acids in mEq. per liter $=$

$\frac{10 \times[(0.80 \times 2)+0.2] \text { lipid phosphorus (mg. \%) }}{31}$

Esterified cholesterol was calculated by subtracting the free cholesterol from the total cholesterol. When free cholesterol was not determined, a ratio of free to total cholesterol of 0.28 , the average ratio of normal sera (4), was assumed in order to estimate the ester. In these instances the spaces under "free cholesterol" and "per cent free cholesterol" in the tables were left blank.

The estimation of neutral fat is subject to the combined errors of the other determinations. It is also based on the assumption that 20 per cent of the total phospholipids carry one fatty acid molecule, and 80 per cent carry two. Deviations from this distribution would contribute further error, but it is unlikely that such deviations are great enough to be of serious consequence. Another assumption which requires verification is that all fatty acids not accounted for as phospholipid or cholesterol ester are present as triglyceride (neutral fat). The possibility that a portion of the calculated "neutral fat" is present
Cholesterol and lipid phosphorus are expressed in $\mathrm{mg}$. per cent while total fatty acids and neutral fat are expressed in mEq. per liter, the terms in which they are actually measured.

In two instances the insoluble lipid was washed free of the subnatant serum by three-fold centrifugation with saline, and the lipid material so obtained was analyzed for lipids and proteins. Proteins were determined by the Kjeldahl method.

\section{RESULTS}

The sera fall into two rather clear-cut groups, designated "clear" and "lactescent." This division was to some extent arbitrary, many of the "clear" group showing the faint opalescence common in normal serum. The category "lactescent" was reserved for those sera having a definite cloudiness. The lipid values for the clear sera are given in Table I and those for the lactescent sera in Table II, with the exception of those diabetics whose serum changed from lactescent to clear while they were under observation. These are listed in Table III to show the chemical changes coincident with recovery from acidosis and clearing of serum.

The clear sera were found in patients with diabetes, liver diseases, obstructive jaundice, xanthomatosis of the hypercholesterolemic type, and hypothyroidism. The chemical findings, shown in Table I, were notable in that the neutral fat was in many instances within the normal range of 0 to $6 \mathrm{mEq}$. per liter (4) and never greater than 15.2 mEq. per liter. The cholesterols and with one exception the phospholipids were elevated, according to standards established in this laboratory (4).

In keeping with the clear appearance of these sera was the almost negligible quantity of insoluble lipid, most of which was neutral fat. Two and one-half $\mathrm{mEq}$. of insoluble neutral fat fatty acids per liter was the greatest concentration that was present without distinctly clouding the serum.

The lipid values of the lactescent sera are given in Tables II and III. The conditions represented in this group were essential hyperlipemia, acute pancreatitis, hyperlipemia secondary to nephrosis

as unesterified or free fatty acids, the presence of which has been reported after heparin administration (5-7), remains as another source of error, but preliminary studies have revealed that free fatty acids do not occur to an appreciable extent in normal serum, in post-prandial lipemia, in lactescent serum, or in chyle. 
or diabetic acidosis, and in one instance (No. 12) chronic alcoholism, the lactescence being noted after an alcoholic bout, and disappearing when alcohol was withdrawn.

While all lipid elements were elevated in the lactescent sera, the neutral fat differentiated this group most strikingly from the group with clear sera. When the neutral fat of the original serum was below $14 \mathrm{mEq}$. per liter, the serum was clear, with the exception of that of No. 22 (Table III), which was slightly lactescent when the neutral fat was only $12.4 \mathrm{mEq}$. per liter. Lactescence appeared variably above $14 \mathrm{mEq}$. per liter, and definitely above $20 \mathrm{mEq}$. per liter of neutral fat. When the neutral fat was $90 \mathrm{mEq}$. per liter or higher, the normal straw color of the serum was completely obscured by an opaque milky appearance. The insoluble material causing the lactescence was composed chiefly of neutral fat, but it also included considerable quantities of cholesterol and phospholipid. As neutral fat rose, increasingly larger proportions of cholesterol and phospholipid were found in the insoluble fraction, though there was no constant relationship between the concentration of either of these and lactescence. The soluble cholesterols in the markedly lactescent sera (neutral fat greater than $90 \mathrm{mEq}$. per liter) were between 90 and $321 \mathrm{mg}$. per cent, though the total cholesterols of these sera were between 526 and $1508 \mathrm{mg}$. per cent. In clear sera the soluble cholesterol was as high as $831 \mathrm{mg}$. per cent.

The free cholesterol was in the normal range

TABLE I

Serum lipids before and after centrifugation: Patients with elevated cholesterol, clear serum

\begin{tabular}{|c|c|c|c|c|c|c|c|c|c|c|}
\hline \multirow[b]{2}{*}{ Patient } & \multirow{2}{*}{$\begin{array}{c}\text { Unit } \\
\text { number } \\
\text { Age Sex }\end{array}$} & \multirow[b]{2}{*}{ Date } & & \multirow[b]{2}{*}{$\begin{array}{l}\text { Total } \\
\text { fatty } \\
\text { acids }\end{array}$} & \multicolumn{3}{|c|}{ Cholesterol } & \multirow{2}{*}{$\begin{array}{c}\text { Lipid } \\
\text { phos- } \\
\text { phorus }\end{array}$} & \multirow{2}{*}{$\begin{array}{c}\text { Neutral } \\
\text { fat } \\
\text { fatty } \\
\text { acid } \\
\end{array}$} & \multirow[b]{2}{*}{ Comment } \\
\hline & & & & & Total & Free & $\frac{\text { Free }}{\text { Total }}$ & & & \\
\hline 1 & $\begin{array}{l}\text { A67585 } \\
36 \quad F\end{array}$ & $3 / 13 / 53$ & $\begin{array}{l}\text { Original } \\
\text { Subnatant } \\
\text { Difference }\end{array}$ & $\begin{array}{c}m E q . / L \\
21.7 \\
20.3 \\
1.4\end{array}$ & $\begin{array}{c}\text { mg. } \% \\
400 \\
386 \\
14\end{array}$ & $\begin{array}{c}m g . \% \\
167 \\
157 \\
10\end{array}$ & $\begin{array}{l}\% \\
42 \\
41 \\
71\end{array}$ & $\begin{array}{c}\text { mg. } \% \\
20.0 \\
19.2 \\
0.8\end{array}$ & $\begin{array}{c}m E q . / L \\
4.0 \\
2.6 \\
1.4\end{array}$ & $\begin{array}{l}\text { Biliary cirrhosis. } \\
\text { Serum icteric }\end{array}$ \\
\hline 2 & $\begin{array}{l}\mathrm{C} 21335 \\
53 \quad \mathrm{~F}\end{array}$ & $4 / 13 / 53$ & $\begin{array}{l}\text { Original } \\
\text { Subnatant } \\
\text { Difference }\end{array}$ & $\begin{array}{r}24.2 \\
20.9 \\
3.3\end{array}$ & $\begin{array}{r}423 \\
378 \\
45\end{array}$ & 116 & 27 & 16.2 & .6 .8 & $\begin{array}{l}\text { Hypopituitary with } \\
\text { hypothyroidism. } \\
\text { Faintly lactescent }\end{array}$ \\
\hline 3 & $\begin{array}{l}\text { C11339 } \\
29 \mathrm{M}\end{array}$ & $2 / 25 / 53$ & $\begin{array}{l}\text { Original } \\
\text { Subnatant } \\
\text { Difference }\end{array}$ & $\begin{array}{r}26.9 \\
24.6 \\
2.2\end{array}$ & $\begin{array}{r}559 \\
535 \\
24\end{array}$ & $\begin{array}{r}162 \\
154 \\
8\end{array}$ & $\begin{array}{l}29 \\
29 \\
33\end{array}$ & $\begin{array}{r}19.7 \\
19.3 \\
0.4\end{array}$ & $\begin{array}{l}5.6 \\
3.6 \\
2.0\end{array}$ & $\begin{array}{l}\text { Hypercholesterolemic } \\
\text { xanthomatosis }\end{array}$ \\
\hline \multirow[t]{2}{*}{4} & $\begin{array}{l}\text { Ros. } \\
40\end{array}$ & $6 / 8 / 53$ & $\begin{array}{l}\text { Original } \\
\text { Subnatant } \\
\text { Difference }\end{array}$ & $\begin{array}{r}22.2 \\
20.6 \\
1.6\end{array}$ & $\begin{array}{r}402 \\
391 \\
11\end{array}$ & 116 & 29 & $\begin{array}{r}12.0 \\
10.5 \\
1.5\end{array}$ & $\begin{array}{l}7.8 \\
7.4 \\
0.4\end{array}$ & $\begin{array}{l}\text { Hypercholesterolemic } \\
\text { xanthomatosis }\end{array}$ \\
\hline & & $10 / 7 / 53$ & $\begin{array}{l}\text { Original } \\
\text { Subnatant } \\
\text { Difference }\end{array}$ & $\begin{array}{r}22.8 \\
21.6 \\
1.2\end{array}$ & $\begin{array}{r}362 \\
317 \\
45\end{array}$ & 106 & 29 & $\begin{array}{r}15.9 \\
15.8 \\
0.1\end{array}$ & $\begin{array}{l}7.0 \\
6.6 \\
0.4\end{array}$ & $\cdot$ \\
\hline 5 & ${ }_{33}^{\text {Ave. }} \mathbf{M}$ & $4 / 21 / 53$ & $\begin{array}{l}\text { Original } \\
\text { Subnatant } \\
\text { Difference }\end{array}$ & $\begin{array}{r}18.8 \\
17.9 \\
0.9\end{array}$ & $\begin{array}{r}394 \\
368 \\
26\end{array}$ & $\begin{array}{r}106 \\
98 \\
8\end{array}$ & $\begin{array}{l}27 \\
27 \\
31\end{array}$ & $\begin{array}{r}12.9 \\
12.4 \\
0.5\end{array}$ & $\begin{array}{l}3.9 \\
3.2 \\
0.7\end{array}$ & $\begin{array}{l}\text { Hypercholesterolemic } \\
\text { xanthomatosis }\end{array}$ \\
\hline 6 & $\begin{array}{l}\text { A66992 } \\
48 \quad \mathrm{~F}\end{array}$ & $1 / 5 / 53$ & $\begin{array}{l}\text { Original } \\
\text { Subnatant } \\
\text { Difference }\end{array}$ & $\begin{array}{r}35.1 \\
30.5 \\
4.6\end{array}$ & $\begin{array}{r}412 \\
389 \\
23\end{array}$ & $\begin{array}{r}121 \\
116 \\
5\end{array}$ & $\begin{array}{l}29 \\
30 \\
22\end{array}$ & $\begin{array}{r}19.0 \\
18.4 \\
0.6\end{array}$ & $\begin{array}{r}15.2 \\
12.7 \\
2.5\end{array}$ & $\begin{array}{l}\text { Diabetic ketosis. } \\
\text { Faintly lactescent }\end{array}$ \\
\hline \multirow[t]{2}{*}{7} & $\begin{array}{l}40-11-26 \\
42 \quad \mathrm{~F}\end{array}$ & $1 / 6 / 54$ & $\begin{array}{l}\text { Original } \\
\text { Subnatant } \\
\text { Difference }\end{array}$ & $\begin{array}{r}45.6 \\
42.6 \\
3.0\end{array}$ & $\begin{array}{r}762 \\
722 \\
40\end{array}$ & 702 & 92 & $\begin{array}{r}57.0 \\
52.0 \\
5.0\end{array}$ & $\begin{array}{r}11.2 \\
11.3 \\
-0.1\end{array}$ & $\begin{array}{l}\text { Biliary cirrhosis. } \\
\text { Serum icteric }\end{array}$ \\
\hline & & $1 / 13 / 54$ & $\begin{array}{l}\text { Original } \\
\text { Subnatant } \\
\text { Difference }\end{array}$ & $\begin{array}{r}48.3 \\
44.5 \\
3.8\end{array}$ & $\begin{array}{r}831 \\
851 \\
-20\end{array}$ & 746 & 90 & 68.1 & 7.2 & \\
\hline 8 & $\begin{array}{l}39-92-70 \\
66 \quad F\end{array}$ & $12 / 2 / 53$ & $\begin{array}{l}\text { Original } \\
\text { Subnatant } \\
\text { Difference }\end{array}$ & $\begin{array}{r}40.8 \\
39.4 \\
1.4\end{array}$ & $\begin{array}{r}555 \\
545 \\
10\end{array}$ & 519 & 93 & $\begin{array}{r}42.8 \\
41.0 \\
1.8\end{array}$ & $\begin{array}{r}14.1 \\
14.3 \\
-0.2\end{array}$ & $\begin{array}{l}\text { Carcinoma of the bile } \\
\text { duct with obstruc- } \\
\text { tive jaundice. Se- } \\
\text { rum icteric }\end{array}$ \\
\hline
\end{tabular}


TABLE II

Serum lipids before and after centrifugation: Patients with lactescent serum

\begin{tabular}{|c|c|c|c|c|c|c|c|c|c|c|}
\hline \multirow[b]{2}{*}{ Patient } & \multirow[b]{2}{*}{$\begin{array}{c}\text { Unit } \\
\text { number } \\
\text { Age }\end{array}$} & \multirow[b]{2}{*}{ Date } & & \multirow[b]{2}{*}{$\begin{array}{l}\text { Total } \\
\text { fatty } \\
\text { acids }\end{array}$} & \multicolumn{3}{|c|}{ Cholesterol } & \multirow[b]{2}{*}{$\begin{array}{l}\text { Lipid } \\
\text { phos- } \\
\text { phorus }\end{array}$} & \multirow{2}{*}{$\begin{array}{l}\text { Neutral } \\
\text { fat } \\
\text { fatty } \\
\text { acid }\end{array}$} & \multirow[b]{2}{*}{ Comment } \\
\hline & & & & & Total & Free & $\frac{\text { Free }}{\text { Total }}$ & & & \\
\hline 9 & $\begin{array}{l}\text { Som. } \\
50\end{array}$ & $10 / 30 / 52$ & $\begin{array}{l}\text { Original } \\
\text { Subnatant } \\
\text { Difference }\end{array}$ & $\begin{array}{c}m E q . / L . \\
56.2 \\
41.8 \\
14.4\end{array}$ & $\begin{array}{r}\text { m8. \% } \\
444 \\
416 \\
28\end{array}$ & mg. $\%$ & $\%$ & $m g . \%$ & $m E q . / L$. & Diabetic acidosis \\
\hline 10 & ${ }_{57}^{16572} \mathrm{~F}$ & $3 / 31 / 53$ & $\begin{array}{l}\text { Original } \\
\text { Subnatant } \\
\text { Difference }\end{array}$ & $\begin{array}{r}234.6 \\
29.7 \\
204.9\end{array}$ & $\begin{array}{l}671 \\
127 \\
544\end{array}$ & $\begin{array}{r}275 \\
54 \\
221\end{array}$ & $\begin{array}{l}41 \\
42 \\
41\end{array}$ & $\begin{array}{l}31.8 \\
10.9 \\
20.9\end{array}$ & $\begin{array}{r}206.5 \\
21.6 \\
184.9\end{array}$ & $\begin{array}{l}\text { Diabetic ketosis with } \\
\text { xanthomatosis }\end{array}$ \\
\hline 11 & $\begin{array}{c}39-38-38 \\
3 \mathrm{M}\end{array}$ & $8 / 21 / 53$ & $\begin{array}{l}\text { Original } \\
\text { Subnatant } \\
\text { Difference }\end{array}$ & $\begin{array}{r}187.0 \\
44.4 \\
142.6\end{array}$ & $\begin{array}{r}1045 \\
280 \\
765\end{array}$ & 271 & 26 & $\begin{array}{l}30.0 \\
17.0 \\
13.0\end{array}$ & $\begin{array}{r}149.6 \\
26.7 \\
122.9\end{array}$ & $\begin{array}{l}\text { Nephrosis } \\
\text { Serum total proteins, } \\
3.30 \mathrm{gm} . \% \\
\text { Albumin, } 0.8 \mathrm{gm} . \% \\
\text { Globulin, } 2.5 \mathrm{gm} . \%\end{array}$ \\
\hline 12 & $\mathrm{Y5}_{45} \mathrm{M}$ & $8 / 20 / 53$ & $\begin{array}{l}\text { Original } \\
\text { Subnatant } \\
\text { Difference }\end{array}$ & $\begin{array}{l}64.2 \\
31.8 \\
32.4\end{array}$ & $\begin{array}{l}457 \\
263 \\
194\end{array}$ & $\begin{array}{l}286 \\
168 \\
118\end{array}$ & $\begin{array}{l}62 \\
63 \\
61\end{array}$ & $\begin{array}{l}32.0 \\
11.0 \\
21.0\end{array}$ & $\begin{array}{l}45.7 \\
21.8 \\
23.9\end{array}$ & Alcoholic fatty liver \\
\hline 13 & $\begin{array}{l}\text { Brys. } \\
40\end{array}$ & $11 / 11 / 53$ & $\begin{array}{l}\text { Original } \\
\text { Subnatant } \\
\text { Difference }\end{array}$ & $\begin{array}{r}226.7 \\
29.3 \\
197.4\end{array}$ & $\begin{array}{l}857 \\
157 \\
700\end{array}$ & $\begin{array}{r}380 \\
58 \\
322\end{array}$ & $\begin{array}{l}44 \\
37 \\
46\end{array}$ & $\begin{array}{r}32.2 \\
9.7 \\
22.5\end{array}$ & $\begin{array}{r}195.6 \\
21.6 \\
174.0\end{array}$ & $\begin{array}{l}\text { Essential hyperlipe- } \\
\text { mia with xantho- } \\
\text { matosis }\end{array}$ \\
\hline 14 & $\begin{array}{l}\mathrm{B} 60586 \\
31 \quad \mathrm{~F}\end{array}$ & $11 / 17 / 53$ & $\begin{array}{l}\text { Original } \\
\text { Subnatant } \\
\text { Difference }\end{array}$ & $\begin{array}{l}51.3 \\
30.5 \\
20.8\end{array}$ & $\begin{array}{l}331 \\
217 \\
114\end{array}$ & 100 & 30 & $\begin{array}{r}19.0 \\
14.9 \\
4.1\end{array}$ & $\begin{array}{l}34.3 \\
17.9 \\
16.4\end{array}$ & Diabetic acidosis \\
\hline \multirow[t]{5}{*}{15} & $\begin{array}{l}\text { B78936 } \\
50 \stackrel{F}{F}\end{array}$ & $2 / 11 / 53$ & $\begin{array}{l}\text { Original } \\
\text { Subnatant } \\
\text { Difference }\end{array}$ & $\begin{array}{r}135.4 \\
17.6 \\
117.8\end{array}$ & $\begin{array}{r}526 \\
90 \\
436\end{array}$ & $\begin{array}{r}195 \\
46 \\
149\end{array}$ & $\begin{array}{l}37 \\
51 \\
34\end{array}$ & $\begin{array}{l}24.2 \\
11.3 \\
12.9\end{array}$ & $\begin{array}{r}113.4 \\
10.0 \\
103.4\end{array}$ & $\begin{array}{l}\text { Essential hyperlipe- } \\
\text { mia }\end{array}$ \\
\hline & & $3 / 26 / 53$ & $\begin{array}{l}\text { Original } \\
\text { Subnatant } \\
\text { Difference }\end{array}$ & $\begin{array}{r}199.1 \\
35.1 \\
164.0\end{array}$ & $\begin{array}{l}808 \\
187 \\
621\end{array}$ & $\begin{array}{r}341 \\
74 \\
267\end{array}$ & $\begin{array}{l}42 \\
40 \\
43\end{array}$ & $\begin{array}{l}31.0 \\
12.0 \\
19.0\end{array}$ & $\begin{array}{r}169.0 \\
25.2 \\
143.8\end{array}$ & \\
\hline & & $3 / 30 / 53$ & $\begin{array}{l}\text { Original } \\
\text { Subnatant } \\
\text { Difference }\end{array}$ & $\begin{array}{r}185.5 \\
31.8 \\
153.7\end{array}$ & $\begin{array}{l}940 \\
179 \\
761\end{array}$ & 325 & 35 & 35.0 & 149.6 & \\
\hline & & $9 / 14 / 53$ & $\begin{array}{l}\text { Original } \\
\text { Subnatant } \\
\text { Difference }\end{array}$ & $\begin{array}{r}140.5 \\
26.4 \\
114.1\end{array}$ & $\begin{array}{l}717 \\
160 \\
557\end{array}$ & & & $\begin{array}{l}23.4 \\
11.5 \\
11.9\end{array}$ & $\begin{array}{r}114.9 \\
17.3 \\
97.6\end{array}$ & $\begin{array}{l}\text { On } 7 / 9 / 53 \\
\text { Serum total proteins, } \\
\quad 6.32 \mathrm{gm} . \% \\
\text { Albumin, } 3.49 \mathrm{gm} . \% \\
\text { Globulin, } 2.83 \mathrm{gm} . \%\end{array}$ \\
\hline & & $1 / 18 / 54$ & $\begin{array}{l}\text { Original } \\
\text { Subnatant } \\
\text { Difference }\end{array}$ & $\begin{array}{r}133.0 \\
27.5 \\
105.5\end{array}$ & $\begin{array}{l}705 \\
202 \\
503\end{array}$ & 176 & 25 & $\begin{array}{l}27.0 \\
12.3 \\
14.7\end{array}$ & $\begin{array}{r}103.0 \\
16.4 \\
86.6\end{array}$ & \\
\hline 16 & $\begin{array}{l}\text { C63179 } \\
35 \quad F\end{array}$ & $12 / 29 / 53$ & $\begin{array}{l}\text { Original } \\
\text { Subnatant } \\
\text { Difference }\end{array}$ & $\begin{array}{l}46.6 \\
31.3 \\
15.3\end{array}$ & $\begin{array}{r}385 \\
294 \\
91\end{array}$ & & & & & $\begin{array}{l}\text { Diabetic acidosis not } \\
\text { fasting }\end{array}$ \\
\hline 17 & ${ }_{55}^{84107} \mathrm{~F}$ & $2 / 22 / 54$ & $\begin{array}{l}\text { Original } \\
\text { Subnatant } \\
\text { Difference }\end{array}$ & $\begin{array}{l}47.7 \\
34.2 \\
13.5\end{array}$ & $\begin{array}{r}456 \\
424 \\
32\end{array}$ & 149 & 33 & $\begin{array}{r}20.0 \\
16.9 \\
3.1\end{array}$ & $\begin{array}{l}28.1 \\
16.9 \\
11.2\end{array}$ & $\begin{array}{l}\text { Kimmelstiel Wilson's } \\
\text { Disease } \\
\text { Serum total proteins, } \\
3.85 \mathrm{gm} . \% \\
\text { Albumin, } 1.12 \mathrm{gm} . \% \\
\text { Globulin, } 2.73 \mathrm{gm} . \%\end{array}$ \\
\hline 18 & $\underset{\mathrm{M}}{\stackrel{40-53-29}{2}}$ & $4 / 1 / 54$ & $\begin{array}{l}\text { Original } \\
\text { Subnatant } \\
\text { Difference }\end{array}$ & $\begin{array}{r}31.0 \\
22.0 \\
9.0\end{array}$ & $\begin{array}{r}279 \\
282 \\
-3\end{array}$ & 88 & 32 & $\begin{array}{r}16.0 \\
12.0 \\
4.0\end{array}$ & $\begin{array}{r}16.8 \\
10.0 \\
6.8\end{array}$ & $\begin{array}{l}\text { Possible nephritis } \\
\text { Serum total proteins, } \\
8.1 \mathrm{gm} . \% \\
\text { Albumin, } 5.0 \mathrm{gm} . \% \\
\text { Globulin, } 3.0 \mathrm{gm} . \%\end{array}$ \\
\hline 19 & $\underset{21}{\text { Ep. }} \quad F$ & $4 / 21 / 53$ & $\begin{array}{l}\text { Original } \\
\text { Subnatant } \\
\text { Difference }\end{array}$ & $\begin{array}{r}37.9 \\
30.7 \\
7.2\end{array}$ & $\begin{array}{r}315 \\
267 \\
48\end{array}$ & $\begin{array}{l}90 \\
77 \\
13\end{array}$ & $\begin{array}{l}29 \\
29 \\
27\end{array}$ & $\begin{array}{r}13.8 \\
11.8 \\
2.0\end{array}$ & $\begin{array}{r}23.1 \\
18.9 \\
4.2\end{array}$ & Acute pancreatitis \\
\hline
\end{tabular}


TABLE III

Serum lipids before and after centrifugation: Patients with lipemia of diabetic acidosis who showed clearing of serum with treatment

\begin{tabular}{|c|c|c|c|c|c|c|c|c|c|c|}
\hline \multirow[b]{2}{*}{ Patient } & \multirow{2}{*}{$\begin{array}{l}\text { Unit } \\
\text { number } \\
\text { Age Sex }\end{array}$} & \multirow[b]{2}{*}{ Date } & & \multirow[b]{2}{*}{$\begin{array}{l}\text { Total } \\
\text { fatty } \\
\text { acids }\end{array}$} & \multicolumn{3}{|c|}{ Cholesterol } & \multirow{2}{*}{$\begin{array}{l}\text { Lipid } \\
\text { phos- } \\
\text { phorus }\end{array}$} & \multirow{2}{*}{$\begin{array}{l}\text { Neutral } \\
\text { fat } \\
\text { fatty } \\
\text { acid }\end{array}$} & \multirow[b]{2}{*}{ Comment } \\
\hline & & & & & Total & Free & $\frac{\text { Free }}{\text { Total }}$ & & & \\
\hline \multirow[t]{2}{*}{20} & $\begin{array}{l}37-94-70 \\
12\end{array}$ & $11 / 4 / 52$ & $\begin{array}{l}\text { Original } \\
\text { Subnatant } \\
\text { Difference }\end{array}$ & $\begin{array}{c}m E q . / L \\
126.0 \\
23.8 \\
102.2\end{array}$ & $\begin{array}{r}\text { mg. \% } \\
636 \\
122 \\
514\end{array}$ & $\begin{array}{c}\text { mg. } \% \\
256 \\
60 \\
196\end{array}$ & $\begin{array}{l}\% \\
40 \\
49 \\
38\end{array}$ & $\begin{array}{l}m g . \% \\
33.5 \\
10.0 \\
23.5\end{array}$ & $\begin{array}{c}m E q . / L \\
96.8 \\
16.4 \\
80.4\end{array}$ & $\begin{array}{l}\text { Diabetic acidosis. } \\
\text { Very lactescent }\end{array}$ \\
\hline & & $11 / 10 / 52$ & $\begin{array}{l}\text { Original } \\
\text { Subnatant } \\
\text { Difference }\end{array}$ & $\begin{array}{r}23.7 \\
21.7 \\
2.0\end{array}$ & $\begin{array}{r}337 \\
291 \\
46\end{array}$ & 104 & 31 & $\begin{array}{r}14.2 \\
11.9 \\
2.3\end{array}$ & $\begin{array}{l}9.3 \\
9.3 \\
0\end{array}$ & After recovery, clear \\
\hline \multirow[t]{2}{*}{21} & $\begin{array}{l}\text { B72326 } \\
30 \quad \mathrm{~F}\end{array}$ & $5 / 12 / 53$ & $\begin{array}{l}\text { Original } \\
\text { Subnatant } \\
\text { Difference }\end{array}$ & $\begin{array}{l}34.4 \\
21.0 \\
13.4\end{array}$ & $\begin{array}{r}282 \\
187 \\
95\end{array}$ & & & & & $\begin{array}{l}\text { Diabetic acidosis. } \\
\text { Moderately lactes- } \\
\text { cent }\end{array}$ \\
\hline & & $5 / 14 / 53$ & $\begin{array}{l}\text { Original } \\
\text { Subnatant } \\
\text { Difference }\end{array}$ & $\begin{array}{r}19.2 \\
16.5 \\
2.7\end{array}$ & $\begin{array}{r}217 \\
219 \\
-2\end{array}$ & 62 & 28 & $\begin{array}{l}9.6 \\
9.2 \\
0.4\end{array}$ & $\begin{array}{l}9.7 \\
7.1 \\
2.6\end{array}$ & After recovery, clear \\
\hline \multirow[t]{8}{*}{22} & $\begin{array}{l}\text { B82609 } \\
16 \quad M\end{array}$ & $\begin{array}{l}8 / 17 / 53 \\
9 \mathrm{PM}\end{array}$ & $\begin{array}{l}\text { Original } \\
\text { Subnatant } \\
\text { Difference }\end{array}$ & $\begin{array}{r}526.0 \\
33.0 \\
493.0\end{array}$ & $\begin{array}{r}1219 \\
115 \\
1104\end{array}$ & 581 & 48 & $\begin{array}{l}53.0 \\
11.0 \\
42.0\end{array}$ & $\begin{array}{r}478.0 \\
26.0 \\
452.0\end{array}$ & $\begin{array}{l}\text { Diabetic acidosis } \\
\text { xanthomatosis } \\
\text { Very lactescent } \\
\text { Not fasting }\end{array}$ \\
\hline & & $\begin{array}{l}8 / 18 / 53 \\
2: 40 \mathrm{PM}\end{array}$ & $\begin{array}{l}\text { Original } \\
\text { Subnatant } \\
\text { Difference }\end{array}$ & $\begin{array}{r}401.0 \\
30.9 \\
370.1\end{array}$ & $\begin{array}{r}1508 \\
200 \\
1308\end{array}$ & $\begin{array}{r}683 \\
95 \\
588\end{array}$ & $\begin{array}{l}45 \\
48 \\
45\end{array}$ & $\begin{array}{l}50.0 \\
11.2 \\
38.8\end{array}$ & $\begin{array}{r}361.0 \\
21.7 \\
339.3\end{array}$ & $\begin{array}{l}\text { Very lactescent } \\
\text { Not fasting }\end{array}$ \\
\hline & & $4: 40$ PM & $\begin{array}{l}\text { Original } \\
\text { Subnatant } \\
\text { Difference }\end{array}$ & $\begin{array}{r}327.9 \\
33.0 \\
294.9\end{array}$ & $\begin{array}{r}1433 \\
187 \\
1246\end{array}$ & 633 & 44 & $\begin{array}{l}41.7 \\
10.8 \\
30.9\end{array}$ & $\begin{array}{r}284.0 \\
24.1 \\
259.9\end{array}$ & $\begin{array}{l}\text { Very lactescent } \\
\text { Not fasting }\end{array}$ \\
\hline & & $8 / 19 / 53$ & $\begin{array}{l}\text { Original } \\
\text { Subnatant } \\
\text { Difference }\end{array}$ & $\begin{array}{r}231.7 \\
44.6 \\
187.1\end{array}$ & $\begin{array}{r}1370 \\
321 \\
1049\end{array}$ & 575 & 42 & $\begin{array}{l}41.0 \\
16.4 \\
24.6\end{array}$ & $\begin{array}{r}187.8 \\
30.4 \\
157.4\end{array}$ & $\begin{array}{l}\text { Very lactescent } \\
\text { Fasting }\end{array}$ \\
\hline & & $8 / 21 / 53$ & $\begin{array}{l}\text { Original } \\
\text { Subnatant } \\
\text { Difference }\end{array}$ & $\begin{array}{r}105.0 \\
37.3 \\
67.7\end{array}$ & $\begin{array}{r}1194 \\
493 \\
701\end{array}$ & $\begin{array}{l}343 \\
187 \\
156\end{array}$ & $\begin{array}{l}29 \\
38 \\
22\end{array}$ & $\begin{array}{l}34.0 \\
17.9 \\
16.1\end{array}$ & $\begin{array}{l}63.3 \\
17.9 \\
45.4\end{array}$ & $\begin{array}{l}\text { Moderately lactes- } \\
\text { cent }\end{array}$ \\
\hline & & $8 / 25 / 53$ & $\begin{array}{l}\text { Original } \\
\text { Subnatant } \\
\text { Difference }\end{array}$ & $\begin{array}{l}33.6 \\
20.7 \\
12.9\end{array}$ & $\begin{array}{l}661 \\
417 \\
244\end{array}$ & 195 & 29 & $\begin{array}{r}15.9 \\
12.5 \\
3.4\end{array}$ & $\begin{array}{r}12.4 \\
5.8 \\
6.6\end{array}$ & $\begin{array}{l}\text { Slightly lactescent } \\
\text { Xanthomata fading }\end{array}$ \\
\hline & & $8 / 28 / 53$ & $\begin{array}{l}\text { Original } \\
\text { Subnatant } \\
\text { Difference }\end{array}$ & $\begin{array}{r}26.6 \\
23.9 \\
2.7\end{array}$ & $\begin{array}{r}516 \\
426 \\
90\end{array}$ & & & $\begin{array}{r}15.9 \\
15.7 \\
0.2\end{array}$ & $\begin{array}{l}7.9 \\
6.7 \\
1.2\end{array}$ & Slightly opalescent \\
\hline & & $8 / 31 / 53$ & & & & & & & & $\begin{array}{l}\text { Serum total proteins, } \\
5.78 \mathrm{gm} . \% \\
\text { Albumin, } 2.73 \mathrm{gm} . \% \\
\text { Globulin, } 3.05 \mathrm{gm} . \%\end{array}$ \\
\hline
\end{tabular}

of 24 to 32 per cent of the total (4) in all of the clear sera in which it was determined except those from patients having definite evidence of liver or biliary disease. In contrast to this, it was common to find 40 to 48 per cent of the cholesterol in the free form in both the soluble and insoluble fractions of the lactescent group, although the patients from whom these sera were obtained had little evidence of liver disease, the common cause for such elevated ratios.
Protein, total fatty acids, and cholesterol of the washed "cream" layer were determined in two of the sera (Table IV). The protein concentrations, 0.34 and $0.11 \mathrm{gm}$. per cent, were small enough to be attributable to the error of the method ( $0.3 \mathrm{gm}$. per cent). Furthermore, the protein was calculated from the total nitrogen, assuming all of the latter to be in the form of protein. If a correction is made for phospholipid nitrogen, estimated from the lipid phosphorus values in 
TABLE IV

Protein and lipid content of washed cream layer

\begin{tabular}{ccccc}
\hline \hline Patient & Date & Protein & Fatty acid & Cholesterol \\
\hline & & $g m . \%$ & $m E q . / L$. & $m g . \%$ \\
10 & $3 / 31 / 53$ & 0.34 & 211 & 435 \\
15 & $2 / 11 / 53$ & 0.11 & 140 & 477 \\
\hline
\end{tabular}

Table II, the protein values become $0.24 \mathrm{gm}$. per cent and $0.05 \mathrm{gm}$. per cent, which in turn constitute 2.5 and 0.8 per cent of the weight of the fatty acids present in the "cream." 8

\section{DISCUSSION}

The partition of lipids between the soluble and insoluble fractions at once reveals that the concentration of neutral fat in serum determines the presence and degree of lactescence. This is shown in Figure 1, where the soluble and insoluble neu-

${ }^{3}$ A molecular weight of 269 is assumed for the fatty acids. tral fat are plotted against the total neutral fat, the insoluble neutral fat serving as an estimate of the degree of lactescence.

The invariable appearance of lactescence when the neutral fat exceeds $20 \mathrm{mEq}$. per liter, suggests that this level represents a physicochemical limit to the concentration of neutral fat in the subnatant serum. This suggestion is strengthened by the constancy of the soluble neutral fat, which was usually present in concentrations close to $20 \mathrm{mEq}$. per liter and which varied only between the extremes of 10 and $30.4 \mathrm{mEq}$. per liter in all of the lactescent sera studied. All further increments of neutral fat, as much as $452 \mathrm{mEq}$. per liter, occurred in the insoluble fraction.

These findings prompt a further hypothesis, that the primary defect responsible for lactescence is not in the clearing mechanism but in the removal of soluble neutral fat from serum. It seems probable that neutral fat is delivered to serum chiefly in the insoluble form, and that its conversion to

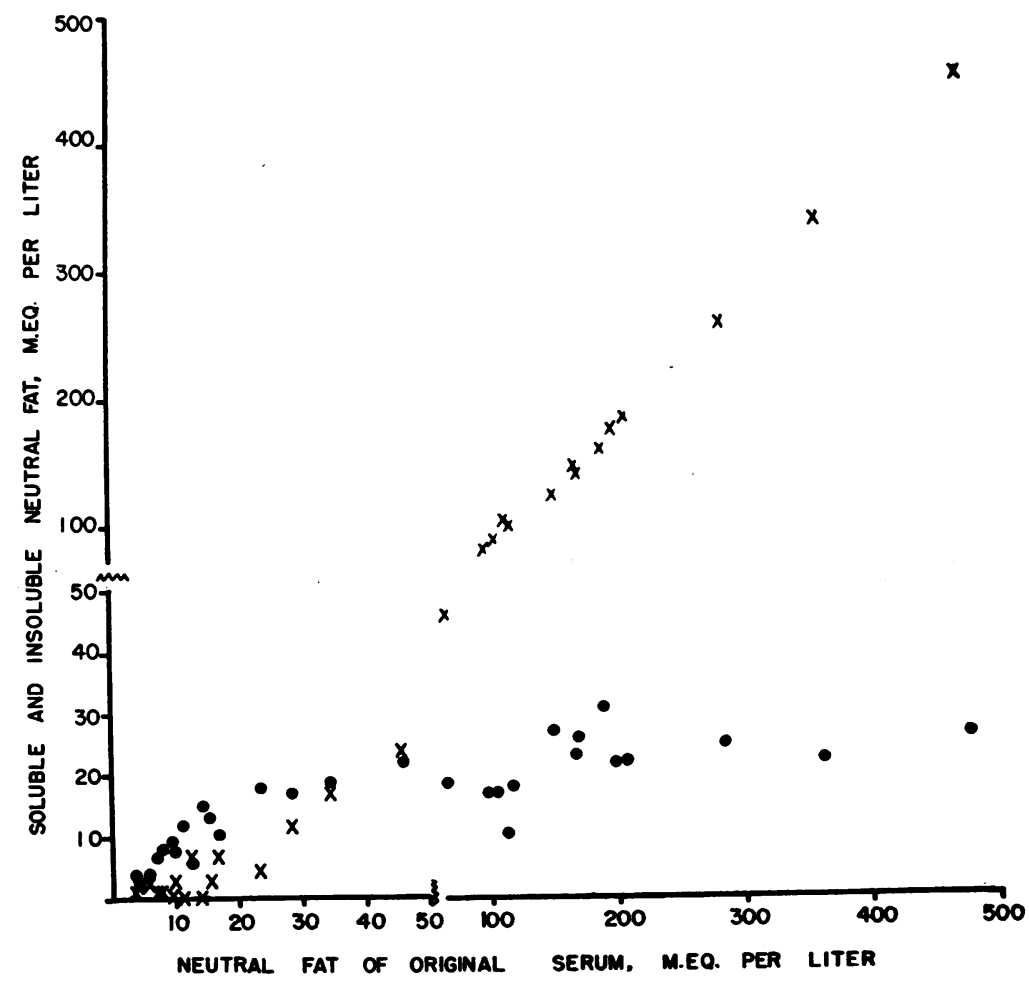

Fig. 1. Relationship of Soluble and Insoluble Neutral Fat to Total Neutral Fat of Original Serum

Soluble neutral fat 0 . Insoluble neutral fat $X$. Note the change in scale above $50 \mathrm{mEq}$. of neutral fat. Neutral fat is expressed as milliequivalents of its constituent fatty acids per liter. 


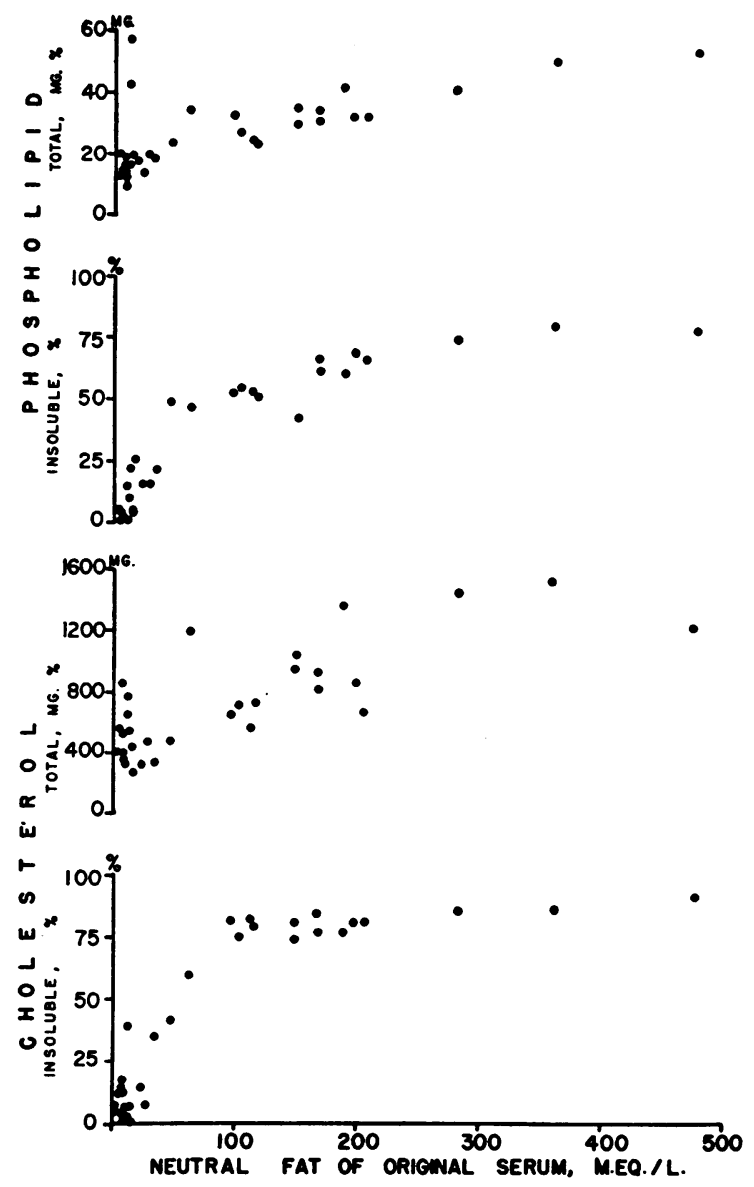

Fig. 2. The Relationship of Neutral Fat to the Total and the Insoluble Cholesterol and PhosPHOLIPID PhOSPHORUS

Cholesterol is plotted both as the total amount in the original serum and as the per cent of the total which was insoluble and rose in the cream layer. Phospholipid phosphorus is charted similarly.

the soluble form is an early step in its metabolism. If there were a block in the removal of soluble neutral fat, the entry of insoluble neutral fat into the soluble fraction could progress only until the limitation noted above was exceeded, after which insoluble neutral fat, denied its normal route of egress, would accumulate, with resultant lactescence. Such a scheme fits well with the data shown in Figure 1.

The presence of cholesterol and phospholipid as well as neutral fat in the "cream" layer may indicate that these substances are dissolved in the neutral fat particles and thereby denied access to the soluble fraction. This would explain the low or normal values of cholesterol and phospholipid in the soluble form, despite their presence in large amounts in the "cream" of lactescent sera (Tables II and III). In Figure 2 both the total concentration of cholesterol and phospholipid, and the relative proportions of each which were in the insoluble fraction, are plotted against the total neutral fat. Not only did cholesterol and phospholipid increase as neutral fat increased, but the relative proportions of each which were found in the insoluble fraction also increased, forming an asymptotic curve when plotted against total neutral fat.

Such a curve could be explained if the insoluble neutral fat were considered the second phase of a two phase system, serum: neutral fat. Any raw cholesterol (or phospholipid) introduced into such a system would then distribute itself between the two phases according to its solubility in each phase. The ratio of the concentration of cholesterol (or phospholipid) in the neutral fat phase to its concentration in the serum phase would be a constant, the distribution coefficient, such as that described by Bischoff, Stauffer, Gray, and Katherman $(8,9)$ for steroid hormones. Such a two phase system, in which the serum phase was of a rather constant quantity in all sera while the size of the neutral fat phase increased with increasing lactescence, would account for the appearance of increasingly greater proportions of cholesterol and phospholipid in the insoluble fraction as neutral fat rose (Figure 2). This does not indicate that cholesterol and phospholipid have exceeded their solubility in serum, but merely that they are more soluble in neutral fat.

By assuming such a two phase system, the distribution coefficient for cholesterol between the neutral fat and the serum phase was calculated for each serum in the following way:

A. Concentration of cholesterol in neutral fat phase $=$ concentration of insoluble cholesterol in mg. per $100 \mathrm{gm}$. insoluble neutral fat. ${ }^{4}$

B. Concentration of cholesterol in aqueous phase $=$ concentration of soluble cholesterol in mg. per $100 \mathrm{ml}$. of serum.

$$
\overline{\mathrm{B}}=\text { distribution coefficient between phases } A
$$
and $B$.

4 Milliequivalents of neutral fat were converted to grams by assuming a molecular weight of 269 for neutral fat fatty acid. 


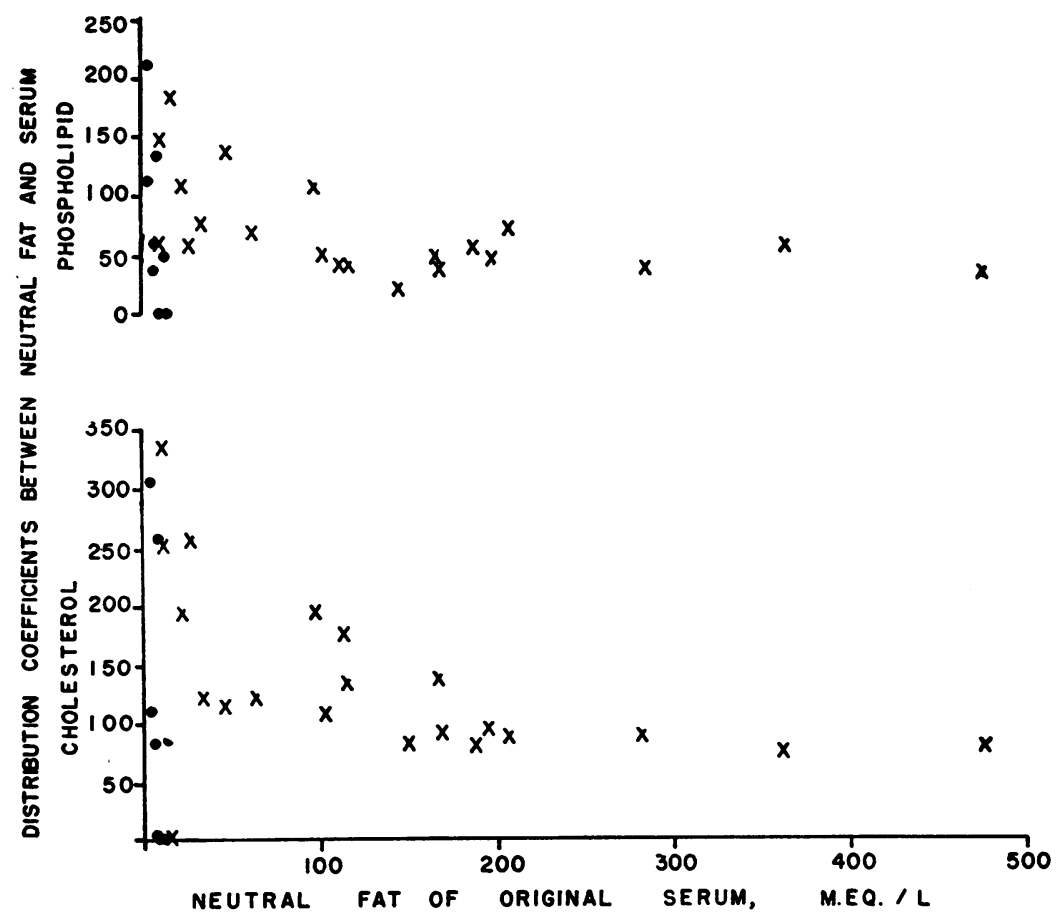

Fig. 3. Distribution Coefficients of Cholesterol and Phospholipto Between Insoluble Neutral Fat and Serum

Clear serum 0 . Lactescent $X$. The coefficients are plotted against the total neutral fat. Two coefficients were too high to be plotted.

Distribution coefficient $=\frac{\begin{array}{l}\text { concentration of insoluble cholesterol (or lipid phosphorus) } \\ \text { in } \mathrm{mg} \text {. per } 100 \mathrm{gm} \text {. insoluble neutral fat. }\end{array}}{\begin{array}{l}\text { concentration of soluble cholesterol (or lipid phosphorus) } \\ \text { in } \mathrm{mg} \text {. per } 100 \mathrm{ml} \text {. clear serum. }\end{array}}$

Similar calculations were carried out for phospholipid. The coefficients for all the sera, both clear and lactescent, are plotted against the total neutral fat in Figure 3. There was great variability at low concentrations where the quantities of insoluble lipid were minute and therefore could not be measured with accuracy, but in those sera having a high neutral fat, the distribution coefficient for cholesterol between insoluble neutral fat and serum was quite constantly about 80 , and that of phospholipid about 50. This suggests that the distribution of cholesterol and phospholipid between the soluble and insoluble fractions depends to some extent on their relative solubility in neutral fat and serum.

Such a hypothetical solubility system, which may account for all the observed lipid characteristics of lactescent serum, may be summarized as follows :
Failure to remove soluble neutral fat from serum $\rightarrow$ accumulation of first soluble, then insoluble neutral fat with resultant lactescence $\rightarrow$ distribution of cholesterol and phospholipid between serum and insoluble neutral fat on a basis of relative solubility.

It is recognized that such a scheme is an oversimplification, since the solubility of lipids per se in aqueous solution is extremely limited. The lipids entering into such true aqueous solution are probably taken up immediately by some substance, probably protein, in combination with which their solubility is greatly increased. The entry of any lipid into the serum fraction would depend both on its solubility in water, and on the avidity with which it is taken up by proteins. In lactescent serum it appears that the proteins cease to remove neutral fat from the aqueous phase, which in turn. 
becomes saturated, with the result that neutral fat accumulates in the insoluble form. Cholesterol and phospholipid, on the other hand, are largely withheld from aqueous solution because of their greater solubility in neutral fat, and are therefore unavailable for lipoprotein formation. Insoluble neutral fat therefore competes with proteins in removing cholesterol and phospholipid from aqueous solution. A physicochemical alteration of serum lipoproteins causing them to be trapped in serum has been proposed by Byers, Friedman, and Rosenman (10) to account for the high cholesterol following the administration of cholic acid and other detergents to rats. It would be of interest to know if the trapping of cholesterol in serum in this case is secondary to an elevation of neutral fat.

The problem of lactescence as outlined above becomes primarily a problem of explaining the limited concentration of neutral fat in the soluble fraction and the failure of neutral fat to be removed from this fraction. It has been assumed that proteins are all-important in maintaining lipids in the soluble state. Normal lipoproteins, though they contain as little as 25 per cent protein in the case of the $\beta$ lipoproteins and 65 per cent in the case of the $\gamma$ lipoproteins, share the physical characteristics of protein, including solubility in water (11, 12). Although the cholesterol and phospholipid content of these lipoproteins has been determined (11-14), little is known of their neutral fat content. Turner and his co-workers (15) presented ultracentrifugal evidence for one or more soluble lipoproteins containing neutral fat, and it is probably safe to conclude that neutral fat owes its solubility to its combination with protein.

In support of the importance of protein in maintaining lipids in the soluble state is the virtual absence of protein from the insoluble lipids of lactescent serum. The protein content of insoluble lipid obtained in the present study was less than 3 per cent by weight (Table IV). Van Eck, Peters, and Man (2) were able to wash the insoluble lipid of postprandial hyperlipemia free of protein. In the chemical method of fractionating lipoproteins (11), a yellow scum obtained after centrifugation is discarded, but the authors state that it probably contains free lipid. Lindgren, Elliott, and Gofman (16) state that the lipid material accumulating at the top of native serum spun for 24 hours at 104,000 g., which included by their classification the Sf 17 and higher particles, contained little or no protein, the quantities found being too small for accurate chemical analysis. Chylomicrons, which could be removed at $9500 \mathrm{~g}$. in 10 minutes, contained nothing but neutral fat and one per cent cholesterol (16). The particles removed by the relatively mild centrifugation of the present study probably included only the larger particles of the Sf 40 class and greater, of minimal protein content. Particles of the Sf 10 to 20 group, which are said to scatter light (17), were probably not affected by the centrifugation, but their presence in the subnatant fraction in more than very small quantities is unlikely judging from the clarity of the "bottom" layer.

Kunkel and Slater (14), using a starch medium for the electrophoretic study of serum lipoproteins, demonstrated that the high neutral fat material of lipemic serum had a mobility similar to $\beta$ lipoprotein. However, for technical reasons these authors were unable to measure neutral fat in the starch medium. Assuming that migration of neutral fat did occur, such a phenomenon could be explained as a loose affinity between globulin and free lipid particles, and does not offer proof that the lipids of such particles are in chemical union with protein. The evidence from the present and other studies favors the conclusion that the particulate lipids which cause lactescence are almost if not completely protein free and have the chemical and physical characteristics of free lipid rather than protein, while the lipids in solution are lipoproteins and owe their solubility to their combination with protein. The limited amount of protein available for lipoprotein formation may well be the critical factor imposing a limit to the concentration of neutral fat in the soluble fraction. Robinson and French (5) demonstrated that the amount of serum albumin present determined the degree of clearing that could be achieved by adding serum from a heparinized rat to chyle. Thoracic duct chyle which in many respects resembles lactescent serum $(18,19)$ also contains both soluble neutral fat and protein, but in concentrations about half as great as those in lactescent serum. Although the protein fractions of the lactescent sera were determined in only a few instances of the present study it is unlikely that albumin alone dictates the level of 


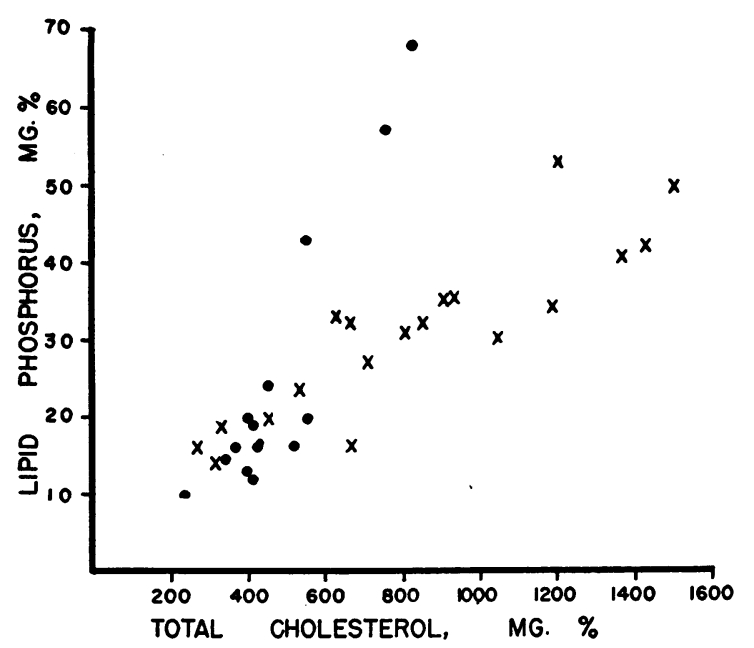

Fig. 4. Relation of Lipid Phosphorus to Total Cholesterol

Clear serum O. Lactescent serum $\times$.

soluble neutral fat. The serum albumin of five patients (Nos. 11, 15, 17, 18, and 22) bore no evident relationship to the soluble neutral fat.

Since proteins are important in maintaining lipids in a soluble state, it may be that an abnormal protein-neutral fat complex in the soluble phase is responsible for the retention of soluble neutral fat in lactescent serum. Abnormal lipoproteins have been reported in a variety of pathological sera, but the specific relationship of these to lactescence is not known because neutral fat is rarely determined.

Repeated allusions appear in the literature suggesting that a disproportionately low phospholipid in relation to cholesterol and total lipid accounts for lactescence of serum, and conversely, a high phospholipid accounts for clarity of serum. This relationship was brought out by Ahrens and Kunkel (3) who compared the clear sera from patients with biliary cirrhosis and jaundice with the lactescent sera from patients with nephrosis, both groups having high total lipids. The high phospholipids in jaundiced patients are statistically related to the high free cholesterol also found in these patients. The relationship between phospholipid and free cholesterol is preserved regardless of whether the ratio of free to total cholesterol is normal $(20,21)$ or elevated (20). The increased ratio of phospholipid to total cholesterol in Ahrens' and Kunkel's (3) patients with biliary cirrhosis is probably merely an indication of the increased ratio of free to total cholesterol in these patients. If the ratios of free to total cholesterol were normal one would expect the phospholipid to total cholesterol ratios also to be normal and of no value in distinguishing between clear and lactescent sera. This was borne out in the present study where there were many sera with normal ratios of free to total cholesterol. The relationship of lipid phosphorus to total cholesterol was similar in both the clear and lactescent sera except for three points which showed a marked deviation above the rest, and represent the only sera with extreme elevations of the ratio of free to total cholesterol (Figure 4). In Figure 5 the relationship of lipid phosphorus to free cholesterol is the same in the clear as in the lactescent sera regardless of the cholesterol ratio. While phospholipids are undoubtedly important constituents of lipoprotein molecules it seems unlikely that a deficit of phospholipid is responsible for lactescence.

An elevation of neutral fat appears to be the only obligatory change necessary for the appearance of lactescence, and is sufficient to account for lactescence regardless of ratios among the other lipid fractions. Because of its limited solubility and its striking influence on the physical state of the other lipids, neutral fat deserves a place of respect in any study of the lipid-protein complexes by which lipids are transported in serum and across cell membranes.

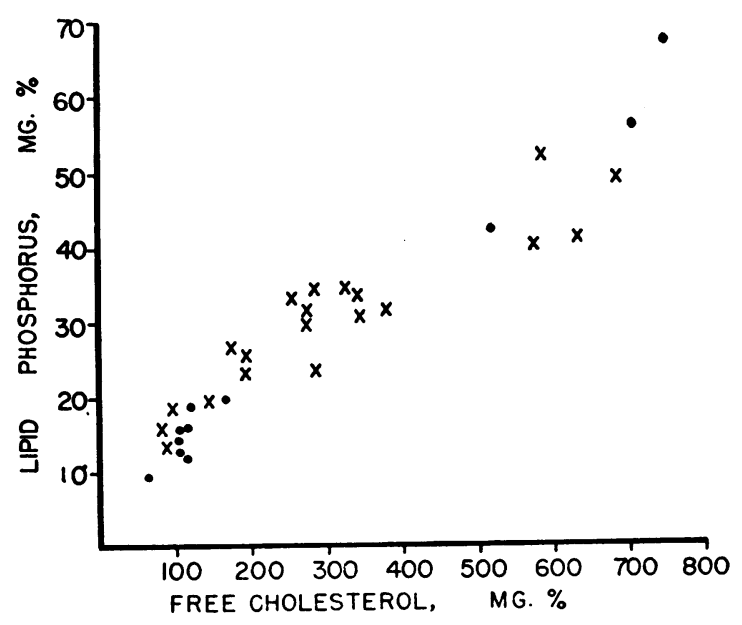

Fig. 5. Relation of Lipid Phosphorus to Free Cholesterol

Clear serum O. Lactescent serum $X$. 


\section{SUM MARY}

1. The state of solubility of serum lipids was measured by comparing serum lipid values before and after removal of insoluble lipids by ultracentrifugation of a variety of clear and lactescent sera having cholesterol values greater than 300 mg. per cent.

2. Neutral fat was found to be the only lipid consistently associated with lactescence. When the neutral fat increased above $20 \mathrm{mEq}$. per liter the serum was invariably lactescent.

3. The soluble neutral fat of lactescent serum was found between the limits of 10 and $30.4 \mathrm{mEq}$. per liter despite variations of total neutral fat from 16.8 to $478 \mathrm{mEq}$. per liter. This was interpreted as indicative of a physiological limit to the concentration of neutral fat in the soluble form.

4. The solubility of phospholipids and cholesterol was decreased when neutral fat increased. The greater solubility of these substances in insoluble neutral fat than in serum is suggested as a possible reason for this relationship.

\section{REFERENCES}

1. Thannhauser, S. J., Lipidoses: Diseases of the cellular lipid metabolism in Oxford Med., Oxford Univ. Press, 1949, vol. IV, part II, chapter VII, p. 214.

2. Van Eck, W. F., Peters, J. P., and Man, E. B., Significance of lactescence in blood serum. Metabolism, 1952, 1, 383.

3. Ahrens, E. H., Jr., and Kunkel, H. G., The stabilization of serum lipid emulsions by serum phospholipids. J. Exper. Med., 1949, 90, 409.

4. Peters, J. P., and Man, E. B., The interrelations of serum lipids in normal persons. J. Clin. Invest., 1943, 22, 707.

5. Robinson, D. S., and French, J. E., The role of albumin in the interaction of chyle and plasma in the rat. Quart. J. Exper. Physiol., 1953, 38, 233.

6. Shore, B., Nichols, A. V., and Freeman, N. K., Evidence for lipolytic action by human plasma obtained after intravenous administration of heparin. Proc. Soc. Exper. Biol. \& Med., 1953, 83, 216.

7. Nichols, A. V., Rubin, L., and Lindgren, F. T., Interaction of heparin active factor and egg-yolk lipoprotein. Proc. Soc. Exper. Biol. \& Med., 1954, 85, 352.

8. Bischoff, F., Stauffer, R. D., and Gray, C. L., Physico-chemical state of the circulating steroids. Am. J. Physiol., 1954, 177, 65.
9. Bischoff, F., and Katherman, R. E., Distribution of estradiol between serum and red cells. Am. J. Physiol., 1948, 152, 189.

10. Byers, S. O., Friedman, M., and Rosenman, R. H., Review: on the regulation of blood cholesterol. Metabolism, 1952, 1, 479.

11. Oncley, J. L., Gurd, F. R. N., and Melin, M., Preparation and properties of serum and plasma proteins. XXV. Composition and properties of human serum beta-lipoprotein. J. Am. Chem. Soc., 1950, 72, 458.

12. Lever, W. F., Gurd, F. R. N., Uroma, E., Brown, R. K., Barnes, B. A., Schmid, K., and Schultz, E. L., Chemical, clinical, and immunological studies on the products of human plasma fractionation. XL. Quantitative separation and determination of the protein components in small amounts of normal human plasma. J. Clin. Invest., 1951, 30, 99.

13. Russ, E. M., Eder, H. A., and Barr, D. P., Proteinlipid relationships in human plasma. I. In normal individuals. Am. J. Med., 1951, 11, 468.

14. Kunkel, H. G., and Slater, R. J., Lipoprotein patterns of serum obtained by zone electrophoresis. J. Clin. Invest., 1952, 31, 677.

15. Turner, R. H., Snavely, J. R., Goldwater, W. H., Randolph, M. L., Sprague, C. C., and Unglaub, W. G., The study of serum proteins and lipids with the aid of the quantity ultracentrifuge. I. Procedure and principal features of the centrifugate of untreated normal serum as determined by quantitative analysis of samples from ten levels. J. Clin. Invest., 1951, 30, 1071.

16. Lindgren, F. T., Elliott, H. A., and Gofman, J. W., The ultracentrifugal characterization and isolation of human blood lipids and lipoproteins, with applications to the study of atherosclerosis. J. Phys. and Coll. Chem., 1951, 55, 80.

17. Gofman, J. W., Lindgren, F., Elliott, H., Mantz, W., Hewitt, J., Strisower, B., and Herring, V., The role of lipids and lipoproteins in atherosclerosis. Science, 1950, 111, 166.

18. Peters, J. P., and Man, E. B., The nature and formation of thoracic duct chyle. Metabolism, 1953, 2, 30.

19. Albrink, M. J., Unpublished observations.

20. Albrink, M. J., Man, E. B., and Peters, J. P., Serum lipids in infectious hepatitis and obstructive jaundice. J. Clin. Invest., 1950, 29, 781.

21. Jackson, R. S., and Wilkinson, C. F., Jr., The ratio between phospholipid and the cholesterols in plasma as an index of human atherosclerosis. Ann. Int. Med., 1952, 37, 1162. 